\title{
To the calculation of the toroidal shell with a local deepening on the external and on the inner surface
}

\author{
Nukh Yakupov ${ }^{1}$, Khakim Kiyamov ${ }^{1}$ and Inzilija Mukhamedova ${ }^{1,2}$ [0000-0001-5869-6686] \\ Institute of Mechanics and Engineering, FRC Kazan Scientific Center, Russian Academy of Sciences, \\ 420111, Kazan , 2/31, Lobachevsky str., Russia \\ Kazan State University of Architecture and Engineering, 420043, Kazan, Zelenaya st., 1, Russia
}

\begin{abstract}
Thin-walled toroidal shells are widely used in the construction During operation, various defects appear on the surface of the shells, in particular, local depressions on the outer and inner surfaces, causing stress concentration in the structure. A three-dimensional spline option of the finite element method was developed to determine the stress-strain state of a toroidal shell with a local deepening on the outer and inner surface. The numerical experiments were carried out. The regularities of the changes in a stress-strain state of the shell with the change in the geometric parameters of the deepening were noted.
\end{abstract}

Keywords: fragment of a toroidal shell, local deepening, threedimensional finite elements, cubic splines, stress concentration.

\section{Introduction}

Toroidal shells are widely used in modern designs. They are used as elements of the structural systems of buildings, engineering constructions, pipeline systems. During operation, under influence of the environment, the physical fields, various defects arise in the structural elements, for example, scratches, dents, local deepenings. In the shells, intended for storage and transportation of the various liquid media, some local corrosion deepenings appear on the inner surface. An increase in stresses in the area of the local deepenings can become a source of destruction [1-2]. This is especially true for thin-walled structures. In this regard, it is of great interest to study distribution of a stress-strain state in the shells with a local change in their geometric parameters, in particular, in the area of local deepenings.

Currently, many studies to assess the stress-strain state of thin-walled structures are carried out on the basis of the universal finite element method (FEM). The idea of a twodimensional spline option of the FEM, combining the idea of parametrizing the middle surface of the entire area under the question and the idea of the finite element method with bicubic approximation of the required variables within each element, was presented in [35]. To assess a stress-strain state of the elements of the thin-walled structures with local blind defects, it becomes necessary to study them in a three-dimensional formulation, in particular, as in [6-13]. 
Calculations of the shells of a complex shape in a two-dimensional formulation by the finite difference method were given in [14]. In the work [15], a method for calculation of toroidal shells, based on an FEM with a break in the middle surface, is considered. In [16] the shells were calculated using a volume octagonal finite element. For a randomly loaded shell of rotation in [17] a hexahedral volume finite element with the unknowns is developed in the form of displacements and stresses. The work [18] presents an algorithm for calculating a structure in the shape of an elliptical cylinder, based on an FEM with interpolation of displacement fields, in which a quadrilateral curvilinear finite element with eighteen degrees of freedom at the node is used.

In this paper, an approach to studying the stress-strain state of a toroidal shell with a local depression located on the inner and outer surfaces of the shell in a three-dimensional formulation is considered and numerical experiments are performed. The regularities of the changes in a stress-strain state of the shell with the change in the geometric parameters of the deepening were noted.

\section{Materials and methods}

\subsection{Basic relations}

A three-dimensional spline option of the FEM combines the idea of parametrizing the entire three-dimensional area and the idea of the finite element method with cubic approximation of the required variables in all three directions within each element.

The three-dimensional area of a fragment of the toroidal shell (Fig. 1) is set by the radius vector

$$
\begin{gathered}
\bar{r}=\left[R_{n}+\left(R_{o}+h z\right) \cos (\beta)\right](\cos \alpha \bar{i}+\sin \alpha \bar{j})+\left(R_{o}+h z\right) \sin (\beta) \bar{k}, \\
\alpha=\frac{\pi t^{2}}{2}, \beta=\pi t^{1}, \quad z=t^{3}, \quad t^{1} \in[-1 ; 1], t^{2} \in[0 ; 1], t^{3} \in[0 ; 1],
\end{gathered}
$$

where Ro is a radius of the circle in a cross section of the shell, $\mathrm{Rn}$ is a distance from the axis of rotation to the center of the generating circle (Fig. 1), $t^{1}, t^{2}, t^{3}$ - coordinates of the unit cube (or parallelepiped).
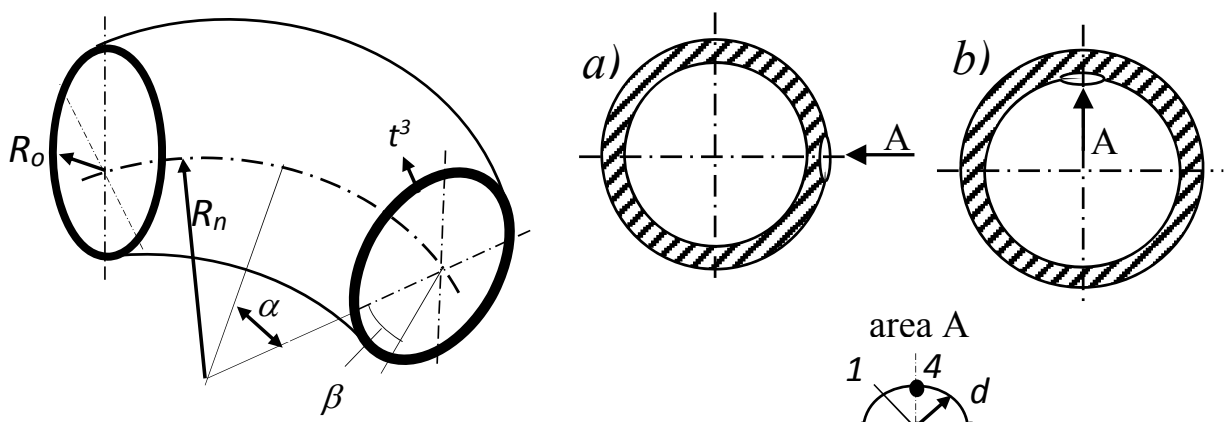

area $\mathrm{A}$

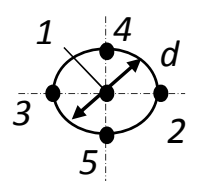

Fig. 1. Fragment of the toroidal shell with a local depression:

a) - on the outer surface $(\alpha=\pi / 4, \beta=\pi)$; b) - on the inner surface. 
In this case, a rectangular grid of the unit cube corresponds to a spatial grid of the entire three-dimensional area under the question.A local depression in a defective elliptical element in plan with semiaxes a and b is set in the form:

$$
\begin{aligned}
h_{k o r}\left(t^{1}, t^{2}\right) & \left.=h_{p} \mathrm{e}^{-\chi}, \quad \chi=\gamma\left[\frac{\left(t^{2}-t_{p}^{2}\right)^{2}}{a^{2}}+\frac{\left(t^{1}-t_{p}^{1}\right)^{2}}{b^{2}}\right)\right], \\
t^{1} & \in[-1 ; 1], t^{2} \in[0 ; 1], t^{3} \in[0 ; 1],
\end{aligned}
$$

where $\gamma$ is a degree of compression-extension of the ellipse along the coordinates $t^{1}$ and $t^{2}$; $t_{p}^{1}, t_{p}^{2}$ are coordinates of the center of the defective area; hp is a minimum thickness of the shell in the defective area at the point $t^{1}=t_{p}^{1}, t^{2}=t_{p}^{2}$.

By differentiating the relations (1) and (2) with respect to $t^{1}, t^{2}, t^{3}$, let us determine the coordinate vectors for the defect-free elements and for the defective ones:

$$
\overline{r_{1}}=\frac{\partial \bar{r}}{\partial t^{1}} ; \quad \overline{r_{2}}=\frac{\partial \bar{r}}{\partial t^{2}} ; \quad \overline{r_{3}}=\frac{\partial \bar{r}}{\partial t^{3}},
$$

covariant components of the metric tensor $g_{i j}$ :

$$
g_{11}=\overline{r_{1} r_{1}}, g_{12}=\overline{r_{1} r_{2}}, g_{22}=\overline{r_{2}} \overline{r_{2}}, g_{13}=\overline{r_{1} r_{3}}, g_{23}=\overline{r_{2}} \overline{r_{3}}, g_{33}=\overline{r_{3}} \overline{r_{3}}
$$

and a discriminant of the metric tensor:

$$
\begin{aligned}
& g=g_{33}\left(g_{11} g_{22}-g_{12}^{2}\right)-g_{32}\left(g_{11} g_{23}-g_{21} g_{13}\right)+ \\
& +g_{31}\left(g_{12} g_{23}-g_{13} g_{22}\right) .
\end{aligned}
$$

Based on (4) and (5), the Christoffel symbols can be determined:

$$
\Gamma_{j k}^{i}=\frac{g^{i t}}{2}\left(\frac{\partial g_{j t}}{\partial t^{k}}+\frac{\partial g_{k t}}{\partial t^{j}}+\frac{\partial g_{j k}}{\partial t^{t}}\right)
$$

The resolving relations are derived from the variational Lagrange equation:

$$
\delta \int_{V} W d V=\int_{V} \rho f^{i} \delta u_{i} d V+\int_{S} p^{i} \delta u_{i} d S
$$

where $W$ is a specific potential energy of deformation of a three-dimensional body; $f^{i}, p^{i}$ are contravariant components of the vector of the mass and surface forces; $\rho$ is a mass density; $u_{i}$ is covariant components of the vector of the required variables; $S$ is a surface of the side faces of the body.

The considered area of the unit cube is devided into finite elements and the solution $u 1$ $=\mathrm{u}, \mathrm{u} 2=$ v и $\mathrm{u} 3=\mathrm{w}$ in each of them is represented in the form of an interpolation cubic spline of three variables [4-5]:

$$
\begin{gathered}
\mathrm{u}=\left[\psi_{1}\left(\mathrm{~s}^{1}\right) \times \psi_{2}\left(\mathrm{~s}^{2}\right) \times \psi_{3}\left(\mathrm{~s}^{3}\right)\right] \otimes \mathrm{F}_{\mathrm{U}}, \\
\mathrm{v}=\left[\psi_{1}\left(\mathrm{~s}^{1}\right) \times \psi_{2}\left(\mathrm{~s}^{2}\right) \times \psi_{3}\left(\mathrm{~s}^{3}\right)\right] \otimes \mathrm{F}_{\mathrm{V}}, \\
\mathrm{w}=\left[\psi_{1}\left(\mathrm{~s}^{1}\right) \times \psi_{2}\left(\mathrm{~s}^{2}\right) \times \psi_{3}\left(\mathrm{~s}^{3}\right)\right] \otimes \mathrm{F}_{\mathrm{W}}, \\
s^{1}=\frac{t^{1}-t_{i}^{1}}{h_{i}^{1}}, s^{2}=\frac{t^{2}-t_{i}^{2}}{h_{j}^{2}}, s^{3}=\frac{t^{3}-t_{i}^{3}}{h_{k}^{3}}, h_{i}^{1}=t^{1}-t_{i}^{1}, h_{j}^{2}=t^{2}-t_{j}^{2}, h_{k}^{3}=t^{3}-t_{k}^{3},
\end{gathered}
$$


where $\psi_{1}, \psi_{2}, \psi_{3}$ are vectors of the coordinate functions along three corresponding coordinate lines, $\mathrm{F}_{\mathrm{U}}, \mathrm{F}_{\mathrm{V}}, \mathrm{F}_{\mathrm{W}}$ are three-dimensional matrices of the components of the required unknowns $\mathrm{u}, \mathrm{v}, \mathrm{w}$ and its derivatives, respectively.

By substituting the variations of displacements and deformations, taking into account the independence of the nodal displacements and their derivatives, after a number of transformations the problem is reduced to a system of algebraic equations of the form:

$$
[A]\{U\}=\{R\},
$$

where $[A]$ is a stiffness matrix of the tape structure system, $\{U\}$ is a vector of the unknowns, $\{R\}$ is a load vector. The structure of all integrals is the same.

\subsection{Example 1}

A fragment of the toroidal shell fixed on the outer surface along both contours is considered. The shell is under the internal pressure $\mathrm{p}=100 \mathrm{~kg} / \mathrm{cm}^{2}$. The shell parameters are: elastic modulus $\mathrm{E}=2100000 \mathrm{~kg} / \mathrm{cm}^{2}$; Poisson's ratio $v=0,3$; thickness $\mathrm{h}=0,9 \mathrm{~cm}$; radii $R_{n}=123 \mathrm{~cm}, R_{0}=41 \mathrm{~cm}$ (Fig. 1). The numerical experiments were carried out for the shell with a local deepening of the diameter $d=1,8 \mathrm{~cm}$ on the outer surface with the depths of the defect $\mathrm{t}=2,4$ and $6 \mathrm{~mm}$.

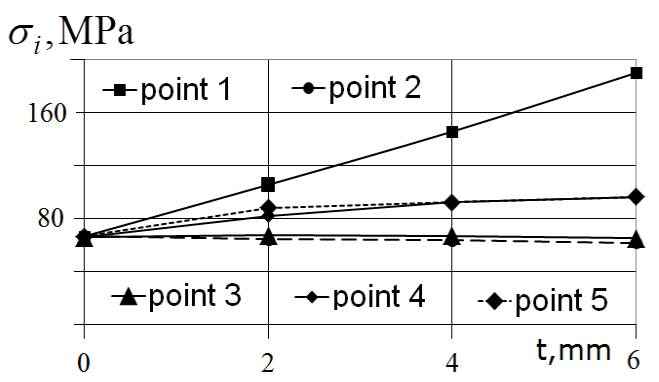

Fig. 2. Dependence of stress intensity $\sigma_{i}$ on changes in depth of defect t.

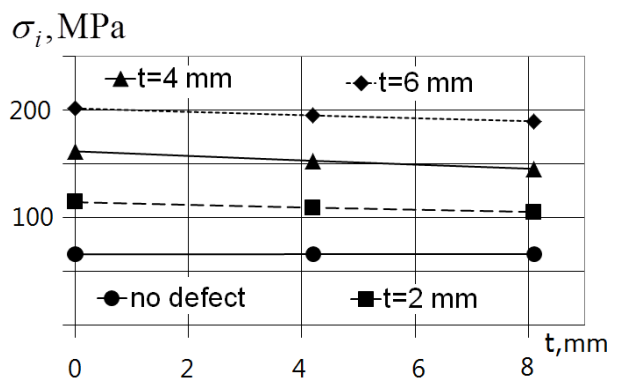

Fig. 3. Change in stress intensity $\sigma_{i}$ over shell thickness $\mathrm{h}$ in the area of point 1.

The shell is divided into 64 elements (144 nodes) - by the thickness $\mathrm{h} \in[0 ; 0,9 \mathrm{~cm}]: t^{3}=$ $0,9\{0 ; 1\}$; by the angle $\alpha \in[0, \pi / 2]: t^{2}=\pi / 2\{0.0,0.125,0.25,0.375,0.5,0.625,0.75,0.875$, $1.0\}$ and by the angle $\beta \in[-\pi,+\pi]: t^{l}=\pi\{-1.0,-0.75,-0.5,-0.25,0.0,0.25,0.5,0.75,1.0\}$.

The dependences of the stress intensity near the outer surface $(\mathrm{t} 3=0.81)$ on the depth of the defect $t$ for the control points 1 to 5 (Fig. 1) are shown in Fig. 2. It was revealed that with increasing the depth of the defect $t$ in the area of the point 1 , as well as in the area of the points 4 and 5 , the stress intensity $\sigma_{i}$ increases to $45 \%$, and in the area of the points 2 and 3 it decreases by $5-7 \%$.

For the area of the point 1 (Fig. 1), the dependence of the change in stress intensity $\sigma_{i}$ over the shell thickness is plotted, starting from the inner surface, for the considered deepenings $t$ (Fig. 3). It can be seen from the figure that as the depth $t$ increases, the stress intensity $\sigma_{i}$ in the center of the defect grows. 


\subsection{Example 2}

A part of the toroidal shell is considered, as in example 1. The numerical experiments were carried out for a shell with a local deepening on the inner surface 1 (Fig. 1b). The deepening diameters were considered: $\mathrm{d}=1.8 \mathrm{~cm} ; \mathrm{d}=2.4 \mathrm{~cm} ; \mathrm{d}=3 \mathrm{~cm}$ at the defect depths $\mathrm{t}=2,4$ and $6 \mathrm{~mm}$.

Fig. 5 shows the dependence of the stress intensity $\sigma_{i}$ with varying the parameter $t^{2}$, which determines the curvature of the shell in the range from $0 \leq t^{2} \leq \pi / 2$ in the area of the defect center.

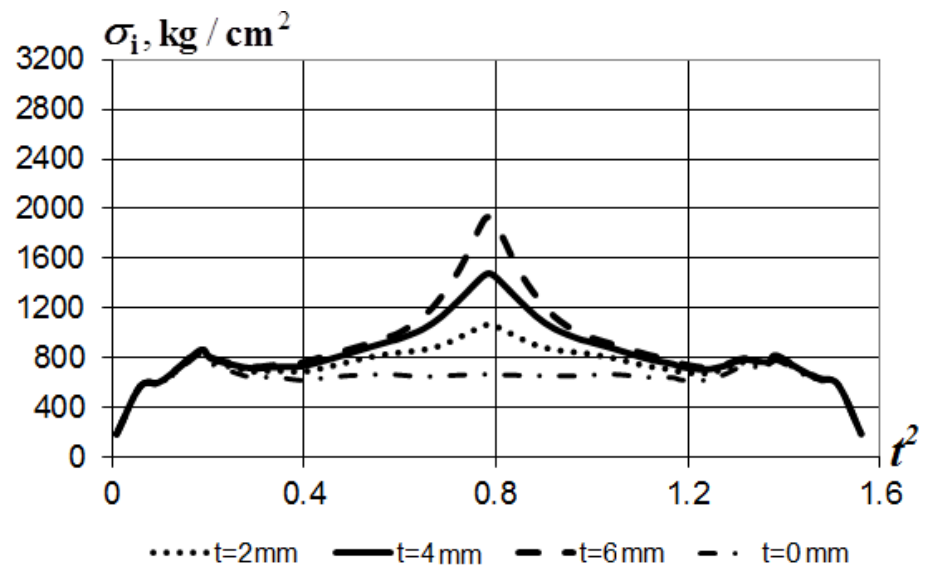

Fig. 4. Dependence of the stress intensity $\sigma_{i}$ on the parameter $t^{2}$ in the range from $0 \leq t^{2} \leq \pi / 2$ $(d=18 \mathrm{~mm} ; h=8.1 \mathrm{~mm})$.

As can be seen from Fig. 4 with an increase in the defect depth, the stress intensity in the defect area increases.

\section{Results}

An effective method for calculating toroidal shells with a local deepening has been developed.

The maximum stress intensity arises in the central part of the deepening.

With an increase in the defect depth, the stress intensity in the defect area increases.

\section{Discussion}

Some numerical experiments were carried out to determine a stress-strain state in the defect area in a form of the local deepening, located on the outer and inner surfaces of the shell, depending on the location and parameters of the deepening.

The regularities of the change in a stress-strain state of the shell with a change in the geometrical parameters of the deepening were established.

\section{References}

1. J. Peng, C. Zhou, J. Xue, Q. Dai, X. He, Safety assessment of pipes with multiple local wall thinning defects under pressure and bending moment, Nucl. Eng. Des., 8 (241), 2758-2765 (2011) 
2. J. W. Kim, C. Y. Park, Effect of length of thinning area on the failure behavior of carbon steel pipe containing a defect of wall thinning, Nucl. Eng. Des., 3 (220), 274284 (2003)

3. N. M. Yakupov, On a method for calculating shells of complex geometry (Research on the theory of shells. Tr. seminar, issue 17, Part II, Kazan, 1984)

4. M. S. Kornishin, N. M. Yakupov, Spline option of the finite element method for calculating shells of complex geometry, Applied Mechanics, 23(3), 38-44 (1987)

5. M. S. Kornishin, N. M. Yakupov, On the calculation of shells of complex geometry in cylindrical coordinates based on the spline option of the FEM, Applied Mechanics, 25(8), 53-60 (1989)

6. N. M. Yakupov, Kh. G. Kiyamov, Sh. Sh. Galyaviev, R. Z. Khisamov, Methods and approaches to the study of a stress-strain state of the structures of complex geometry, Construction. News of universities, 8(524), 14-18 (2002)

7. N. M. Yakupov, Kh. G. Kiyamov, S. N. Yakupov, I. Kh. Kiyamov, Modeling of structural elements of complex geometry by three-dimensional finite elements, Mechanics of composite materials and structures17(1), 145-154 (2011)

8. N.M. Yakupov, H.G. Kiyamov, S.N. Yakupov. Modelling of cyclic shells with complex geometry three-dimensional finite elements, Journal of Physics: Conference series, 1158 (2019) DOI: 10.1088/1742-6596/1158/4/042038.

9. N. M. Yakupov, Kh. G. Kiyamov, Sh. Sh. Galyaviev, R. Z. Khisamov, Methods and approachesto the study of the stress-strain state of structures of complex geometry, Stroit., Izv. VUZov, 8 (524), 14-18 (2002)

10. S. N. Yakupov, T. R. Nasibullin, Analysis of stress concentration in thin-walled structural elements with a local deepening, Struct. Mech. of Eng. Constr. and Build., 1, 30-36 (2016)

11. S. P. Ivanov, O. G.Ivanov, R. A. Kayumov, I. Z. Mukhamedova, Stress-strain state of physically nonlinear shells with ends embedded in stationary arrays and interacting with an elastic medium, Bulletin of Kazan Technological University, 17(10), 221-225 (2014)

12. O. A. Ammosova, R. A. Kayumov, I. Z. Mukhamedova, Modeling the thermal process when welding polyethylene pipes at naturally low temperatures, Bulletin of Kazan Technological University, 17(9), 71-76 (2014)

13. N. M. Yakupov, H. G. Kiyamov, I. Z. Mukhamedova, Simulation of toroidal shell with local defect, Lobachevskii Journal of Mathematics, 41(7), 1310-1314 (2019) DOI: $10.1134 / \mathrm{S} 1995080220070434$

14. V. I. Gulyaev, V. A. Bazhenov, E. A. Gotsulyak, V. V. Gaidaychuk, Calculation of shells of complex shape (Budivelnyk, Kiev, 1990)

15. A. M. Maslennikov, D. V. Gamilov, Stress-strain state of toroidal shells with fractures of the middle surface, Vestn. of TSASU, 1, 90-93 (2007)

16. A. P. Nikolaev, A. P. Kiselev, To the calculation of shells based on the nite element method, Bulletin of Peoples' Friend. Univ. of Russia, Ser. Eng. Research, 107-111 (2002)

17. N. A. Gureeva, Yu. V. Klochkov, A. P. Nikolaev, Calculation of a shell of rotation under an arbitrary loading using the FEM based on the Reisner functional, Comput. Technol., 13 (4), 51-19 (2008)

18. Yu. V. Klochkov, A. P. Nikolaev, T. A. Kiseleva, Stress-strain state of an elliptical cylinder with an ellipsoidal bottom made of dissimilar materials based on FEM, Izv. Sarat. un-that. New Ser., Ser. Math., Mech. Comput. Sci., 13 (3), 65-70 (2013)

19. R. A. Kayumov, D. E. Strakhov, Prediction of deformation in time of polymeric materials with shape memory at different temperatures, Izvestija KGASU, 2 (16), 195-199 (2011) 
20. R. A. Kayumov, D. E. Strakhov, The study of the rheological properties of composite materials of power elements of building structures, IOP Conference Series: Materials Science and Engineering, 890, (2020) DOI: 10.1088/1757-899X/890/1/012096

21. R. A. Kayumov, I. Z. Muhamedova, V. V. Khammatova, Analysis of influence of cold plasma on stiffness properties of polymeric materials, IOP Conference Series: Materials Science and Engineering, 890, (2020) DOI: 10.1088/1757899X/890/1/012092 